\section{Nucleotide Sequence of Fortimicin KL1 Methyltransferase Gene Isolated from Micromonospora olivasterospora, and Comparison of Its Deduced Amino Acid Sequence with Those of Methyltransferases Involved in the Biosynthesis of Bialaphos and Fosfomycin}

Sir:

Cobalamin is one of the largest and most complicated enzyme cofactors. Derivatives of vitamin $\mathbf{B}_{12}$ or cobalamin serve as prosthetic groups for several enzymes catalyzing methyl transfer reactions such as methionine synthase $^{1)}$.

During biosynthetic studies on the herbicide bialaphos produced by Streptomyces hygroscopicus SF-12932), we proved that the most characteristic structural feature of bialaphos, i.e. the $\mathrm{C}-\mathrm{P}-\mathrm{C}$ bond, is formed via $\mathrm{P}$ methylation of $\mathrm{N}$-acetyldemethylphosphinothricin catalyzed by $\mathrm{P}$-methyltransferase ${ }^{3)}$. In contrast to ubiquitous $\mathrm{O}-, \mathrm{N}-$ and $\mathrm{C}$-methyltransferases that use S-adenosylmethionine as the methyl donor, this unique enzyme utilizes methylcobalamin as the direct methyl donor.

Recently our biosynthetic studies on the antibiotic fosfomycin produced by Streptomyces wedmorensis

Fig. 1. Three methylation reactions by (A) P-methyltransferase, (B) phosphonoacetaldehyde methyltransferase and (C) fortimicin KL1 methyltransferase.
(A)<smiles>CC(CCOc1ccccc1)C(=O)O</smiles>
$\mathrm{N}-\mathrm{ACDMPT}$

(B)

$$
\begin{aligned}
& \mathrm{PO}_{3} \mathrm{H}_{2} \\
& \mathrm{CH}_{2} \\
& \mathrm{CH}_{2} \\
& \mathrm{C}=\mathrm{O} \\
& \mathrm{H}
\end{aligned}
$$$$
\text { PnAA }
$$

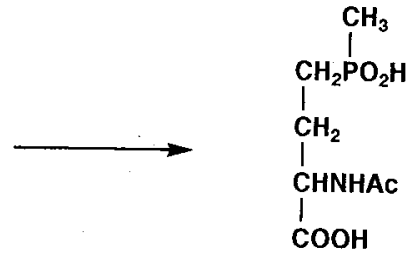

N-AcPT
(C)

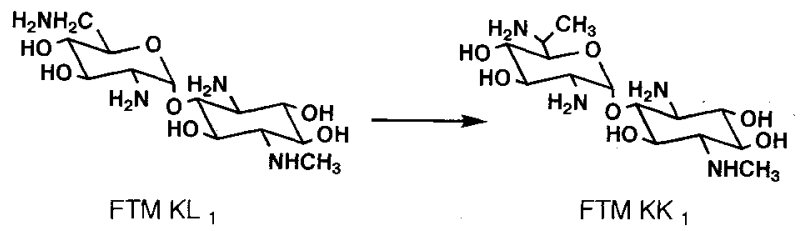

$\mathrm{N}$-AcDMPT $=\mathrm{N}$-acetyldemethylphosphinothricin, $\mathrm{N}$-AcPT $=\mathrm{N}$-acetylphosphinothricin, PnAA = phosphonoacetaldehyde, HPP $=(2 S)$-hydroxypropylphosphonic acid, $\mathrm{FTM}=$ fortimicin . revealed a similar reaction mechanism involving methylcobalamin, i.e. methylation of the aldehyde carbon of phosphonoacetaldehyde (PnAA) to form ( $2 S$ )-hydroxypropylphosphonic acid (Fig. 1 (B) $)^{4)}$. This reaction is catalyzed by PnAA methyltransferase. Based on the finding that fosfomycin non-producing mutants defective in the biosynthesis of vitamin $B_{12}$ incorporated [methyl${ }^{14} \mathrm{C}$ ]methylcobalamin to fosfomycin, we proposed that PnAA was directly methylated by a nucleophilic attack of the methyl anion derived from methylcobalamin catalyzed by PnAA methyltransferase ${ }^{5 \text {. }}$.

More recently we have determined the nucleotide sequences of the genes encoding P-methyltransferase ${ }^{6)}$ and PnAA methyltransferase (GSDB, DDBJ, EMBL and NCBI accession No. D38561) of $S$. hygroscopicus and $S$. wedmorensis, respectively. The deduced amino acid sequences of these two enzymes showed homology to the amino acid sequence of magnesium-protoporphyrin monomethylester oxidative cyclase (BCHE) $)^{7)}(19.9 \%$ identity in 327 amino acids overlap with P-methyltransferase and $24.8 \%$ in 428 amino acids overlap with PnAA methyltransferase). Furthermore these methyltransferases exhibited $18.7 \%$ identity in 374 amino acids overlap each other.

In the biosynthesis of fortimicin (FTM) A, an aminoglycoside antibiotic produced by Micromonospora olivasterospora, an intermediate FTM KL1 is methylated to form FTM KK1 (Fig. 1 (C)) by FTM KL1 methyltransferase ${ }^{8}$. Incorporation experiments with [methyl$\left.{ }^{3} \mathrm{H}\right]$ methylcobalamin and $\left[\right.$ methyl $\left.-{ }^{14} \mathrm{C}\right]$ methionine could not identify the direct methyl donor of FTM KL1 because of high rate incorporations of both radioactive compounds ${ }^{9}$.

In order to reveal the direct methyl donor of FTM KL1 and to obtain information on the reaction mechanism of the methylation, we tried to compare the amino acid sequence deduced from the nucleotide sequence of FTM KLl methyltransferase gene with those of other methyltransferases which utilize methylcobalamin as a methyl donor. The relative location of FTM KL1 methyltransferase gene ( $f m s 7)$ in the FTM biosynthetic gene cluster had been mapped on the basis of complementation experiments described previously (Fig. $2)^{8)}$. The nucleotide sequence of $f m s 7$, however, remained undetermined.

Fig. 2. Organization of the fortimicin biosynthetic gene cluster and plasmids used for sequencing $f m s$.

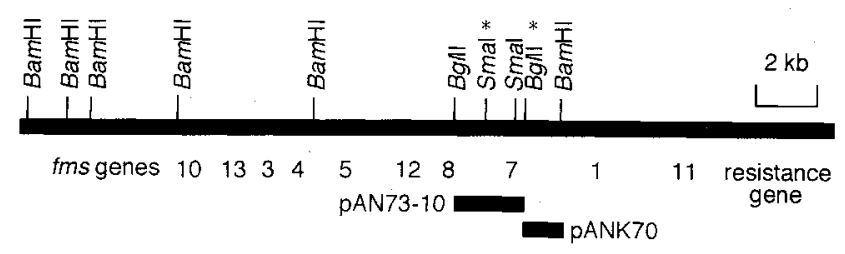

Asterisks represent the restriction endonuclease cutting sites described in text. 
Fig. 3. Homology between the amino acid sequences of fortimicin KL1 methyltransferase (FTMT), phosphonoacetaldehyde methyltransferase (PAMT), P-methyltransferase (PMET) and magnesium-protoporphyrin monomethylester oxidative cyclase (BCHE).

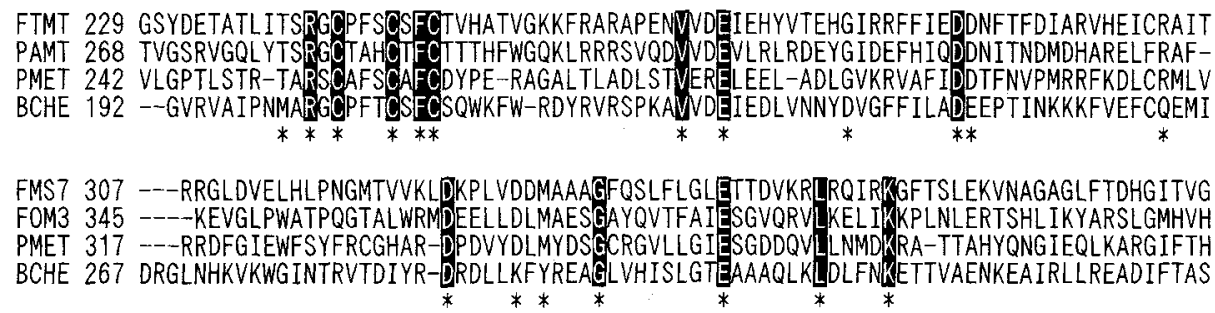

White letters on black background and asterisks indicate the conserved amino acids between four enzymes and between three methylation enzymes, respectively.

In order to reveal the primary structure of FTM KL1 methyltransferase, the nucleotide sequence of a $1412 \mathrm{bp}$ SmaI-BglII fragment in a plasmid pAN73-10 ${ }^{8)}$ (Fig. 2) was obtained on an automated sequencer (LI-COR model $4000 \mathrm{~L}$ ). A partial open reading frame with high $\mathrm{G}+\mathrm{C}$ content in the third position was detected and the deduced amino acid sequence shows $26.0 \%, 19.9 \%$ and $20.7 \%$ homologies to P-methyltransferase, PnAA methyltransferase and BCHE, respectively. Since this open reading frame lacked the $\mathrm{N}$-terminal portion of the protein, an additional $1244 \mathrm{bp} B g l \mathrm{II}-$ Bam HI fragment in a plasmid pANK70 (Fig. 2) was sequenced.

The combined nucleotide data revealed that the $f m s 7$ (GSDB/DDBJ/EMBL/NCBI databases, Accession No. D49442) was encoded by an open reading frame of $1662 \mathrm{bp}$. In addition, a putative Streptomyces ribosome binding site (AAGGG) is seen at the position $13 \mathrm{bp}$ upstream from the initiation codon GTG.

A search of the SWISS-PROT protein sequence database indicated a $24.0 \%$ identity in 383 amino acids overlap between the deduced sequences of FTM methyltransferase and BCHE. FTM methyltransferase exhibited $26.1 \%$ and $23.4 \%$ identities to PnAA methyltransferase and P-methyltransferase, respectively. The similar alignment of the central regions of these three methyltransferases suggests that the reaction mechanisms of these enzymes are similar in utilizing methylcobalamin as the methyl donor. On the other hand, since BCHE catalyzes a quite different chemical reaction, the homology between these three methyltransferases and BCHE presumably results from the structural similarity between methylcobalamin and protoporphyrin. Conserved amino acids among these four enzymes shown in Fig. 3 may participate in the binding of methylcobalamin or protoporphyrin to the enzymes, or in the recognition of these cofactors.

As reported previously, both $\left[\right.$ methyl- $\left.{ }^{3} \mathrm{H}\right]$ methylcobalamin and $\left[\right.$ methyl $\left.-{ }^{14} \mathrm{C}\right]$ methionine were well incorporated into FTM A with the latter being at a higher yield. This result could be interpreted as follows. In the incorporation experiments with these labeled precursors carried out under normal fermentation or in the intact cell system, methylcobalamin might not serve well as a methyl donor presumably due to its instability or poor permeability into the cells. In contrast, methionine, a stable and small molecule, could be incorporated into FTM A more efficiently than methylcobalamin after conversion to the direct precursor, methylcobalamin via $\mathrm{S}$-adenosylmethionine in the cells. Our results obtained by comparison of the amino acid sequences of three methyltransferases clearly corroborated that FTM methyltransferase utilizes methylcobalamin as a direct methyl donor.

These three methyltransferases did not exhibit any significant sequence similarity to methionine synthase that utilizes methylcobalamin as the prosthetic group ${ }^{11}$, suggesting that they catalyze unique reactions different from that of methionine synthase.

\section{Acknowledgments}

Through the courtesy of National Institute of Genetics, protein sequence similarity searching of data bases was carried out by FASTA program.

We thank Kyowa Hakko Kogyo Co., Ltd. for providing us with plasmids used for sequencing $f m s 7$.

This work was supported in part by a Grant-in-Aid for Scientific Research, the Ministry of Science and Culture, Japan (06660129 to T. H.).

TOMOHISA KuZUYama

TaKayuki Seki

TOHRU DAIRI ${ }^{\dagger}$

TOMOMI HIDAKA

HARUo SETO*

Institute of Molecular and Cellular Biosciences,
The University of Tokyo,
Bunkyo-ku, Tokyo 113, Japan
'Biotechnology Research Center,
Faculty of Engineering,
Toyama Prefectural University,
Kurokawa 5180, Kosugi, Toyama 939-03, Japan

(Received May 9, 1995) 


\section{References}

1) Drennan, C. L.; S. Huang, J. T. Drummond, R. G. Matthews \& M. L. LudwiG: How a protein binds $B_{12}$ : A $3.0 \AA \mathrm{X}$-ray structure of $\mathrm{B}_{12}$-binding domains of methionine synthase. Science 266: $1669 \sim 1674,1994$

2) SETo, H.: Biosynthesis of natural C-P compounds, phosphinothricin and bialaphos. In Progress and Prospects of Organophosphorus Agrochemicals. Eds., M. Eto \& J. E. Casida, pp. 179 190, Kyushu University Press, 1995

3) Kamigiri, K.; T. Hidaka, S. Imai, T. Murakami \& H. SETO: Studies on the biosynthesis of bialaphos (SF-1293). 12. C-P bond formation mechanism of bialaphos: Discovery of a P-methylation enzyme. J. Antibiotics 45: 781 787, 1992

4). Seto, H.; T. Hidaka, T. Kuzuyama, S. Shibahara, T. Usui, O. Sakanaka \& S. ImaI: Studies on the biosynthesis of fosfomycin. 2. Conversion of 2-hydroxypropylphosphonic acid to fosfomycin by blocked mutants of Streptomyces wedmorensis. J. Antibiotics 44: 1286 1288, 1991
5) Kuzuyama, T; T. Hidaka, K. Kamigiri, S. Imai \& H. SETO: Studies on the biosynthesis of fosfomycin. 4 . The biosynthetic origin of the methyl group of fosfomycin. J. Antibiotics 45: 1812 1814, 1992

6) Hidaka, T.; M. Hidaka, T. Kuzuyama \& H. Seto: Sequence of a P-methyltransferase-encoding gene isolated from a bialaphos-producing Streptomyces hygroscopicus. Gene 158: $149 \sim 150,1995$

7) Burke, D. H.; M. Alberti, G. A. Armstrong \& J. E. HeArst: The amino acid sequence data has been registered in the EMBL, GenBank and DDBJ data banks with the accession number P26168

8) Dairi, T.; T. Ohta, E. Hashimoto \& M. Hasegawa: Organization and nature of fortimicin A (astromicin) biosynthetic genes studied using a cosmid library of Micromonospora olivasterospora DNA. Mol. Gen. Genet. 236: 39 48, 1992

9) Okumura, S.; T. Deguchi \& H. Marumo: Biosynthetic incorporation of methyl group into fortimicins. J. Antibiotics 34: 1360 1362, 1981 